\title{
A Heterogeneous Multi-scale Model for Blood Flow
}

\author{
Benjamin Czaja $^{1(\otimes)} \mathbb{D}$, Gábor Závodszky ${ }^{1,2}$ (D), and Alfons Hoekstra ${ }^{1}$ (D) \\ 1 Computational Science Lab, Informatics Institute, University of Amsterdam, \\ Amsterdam, Netherlands \\ B.E.Czaja@uva.nl \\ 2 Department of Hydrodynamic Systems, Budapest University of Technology \\ and Economics, Budapest, Hungary
}

\begin{abstract}
This research focuses on developing a heterogeneous multiscale model (HMM) for blood flow. Two separate scales are considered in this study, a Macro-scale, which models whole blood as a continuous fluid and tracks the transport of hematocrit profiles through an advection diffusion solver. And a Micro-scale, which computes directly local diffusion coefficients and viscosities using cell resolved simulations. The coupling between these two scales also includes the use of a surrogate model, which saved local viscosity and diffusion coefficients from previously simulated local hematocrit and shear rate combinations. As the HMM model progresses fewer micro models will be spawned. This is accomplished through the surrogate by interpolating from previously computed viscosities and diffusion coefficients. The benefit of using the HMM method for blood flow is that it, along with resolving the rheology of whole blood, can be extended with other types computational models to model physiological processes like thrombus formation.
\end{abstract}

Keywords: Multi-scale $\cdot$ Blood flow $\cdot$ Rheology

\section{Introduction}

The primary function of the human red blood cell (RBC) is to deliver oxygen to the tissues of the body. From a physiological perspective there are multiple scales at which a red blood cell interacts with the human body. On the largest scales is a healthy human, typical size $\approx 1-2 \mathrm{~m}$, which is maintained though the constant supply of oxygen via the oxygen diffusion from the red blood which occurs on scales of $\mu \mathrm{m}$. The physiological processes that maintains a healthy oxygenated human being is a connection of multiple heterogeneous systems that exists and operate on multiple spatial scales. Mutli-scale computational models are being developed in order to understand how these individual processes are coupled together to maintain a working healthy human being $[17,18]$.

The CompBioMed2 project grant agreement No. 823712.

(C) Springer Nature Switzerland AG 2020

V. V. Krzhizhanovskaya et al. (Eds.): ICCS 2020, LNCS 12142, pp. 403-409, 2020.

https://doi.org/10.1007/978-3-030-50433-5_31 
From a rheological perspective whole blood, which travels the through the cardiovascular system, is a multi-scale process in itself. On the smallest scales $(\mu \mathrm{m})$, are the deformations of a RBC and the corresponding interactions with the suspending blood plasma. Because of the cell nature of whole blood, typically dominated by the deformable RBCs, blood flow on scales below $300 \mu \mathrm{m}$ exhibits non-Newtonian Behavior. Such a hallmark of this behavior is the FåhraeusLindqvist effect [13], which is the decrease in the relative apparent viscosity of whole blood that has been contributed by the lubrication layer on the vessel wall provided by the existence of a red blood cell-free layer (CFL) [14]. Migration of RBCs away from the vessel wall has been since observed in multiple in vitro studies, and has been shown to create two phases over the cross section of a tube; a central region of mainly RBCs, and an on average a cell depleted region, the CFL [10]. The shear thinning behavior of whole blood however is not only limited to the Fåhraeus-Lindqvist effect and may not only occur on the microvascular scale. There are also prominent shear thinning effects of whole blood and has been found to be affected by many things; such as the break up of rouleaux structures [6], RBC alignment, and RBC stretching in flow $[11,16]$. These phenomena have given rise to the fact that whole blood viscosity is also dependent on shear rate [9].

In order to properly resolve the non-Newtonian phenomena, cell resolved blood flow models are required [5,14,27], as they account for the mechanical deformations of the red blood cells and the subsequent influence on the suspension rheology. The transport of other blood cells types on these scales are also influenced by the presence of red blood cells such as the margination of platelets [28] and white blood cells [15] to the vessel wall. Currently numerical models that adequately resolve the transport and rheological properties of whole blood have large amounts of computational overhead and require high performance computing $[3,23]$.

Blood flow on scales larger than $300 \mu \mathrm{m}$ is consistently modeled as a continuous fluid, as it is computationally more convenient because models no longer include the individual cell dynamics [20,24,25], which greatly reduces the computational overhead. Continuous models either assume whole blood as a Newtonian fluid on larger scales or use a non-Newtonian blood viscosity model to approximate the departure of whole from the Newtonian description. Non-Newtonian models describe the change in blood viscosity with a dependency either on shear rate like a power law fluid [22] or Carreau-Yasuda [2], or depends on yield stress like the Casson model [19]. Since such models do not include the dynamics of the cells they may over estimate the transport behaviors which are a result of cell-cell collisions within whole blood suspensions. This may lead to an invalid description of particle diffusivities within whole blood.

In order to capture both the non-Newtonian viscosity change of whole blood along with the proper treatment of the transport of suspended blood cells a multi-scale model must be developed in order to correctly account for both processes on all scales of the cardiovascular system. Because systems of the body are heterogeneous across multiple scales present in the body we chose to 
develop a heterogeneous multi-scale model (HMM) for blood flow. Though this research is focused primarily on the rheology and transport properties of blood, HMM is a more general method for modeling multi scale problems and can be useful for future development as it can incorporate model beyond strictly fluid dynamic problems.

\section{Heterogeneous Multi-scale Model}

The Heterogeneous Multi-scale Method [1] is a modeling technique used to numerically solve multi-scale problems by coupling multiple sub-models together that each solve a component separately, but by combining each separate submodel together an overall macro model emerges. HMM relies on efficient coupling between macro and micro models [12]. A macro scale model can be either limited or too computational expensive to numerical solve an entire problem on its own; therefore micro models are employed to resolve each component of the problem separately and return the result to the macro model. Heterogeneous here suggests the problem is multi-physics in nature [8]. Scale separation is a common exploitation in HMM as many numerical problems are difficult to capture solely with one single model.

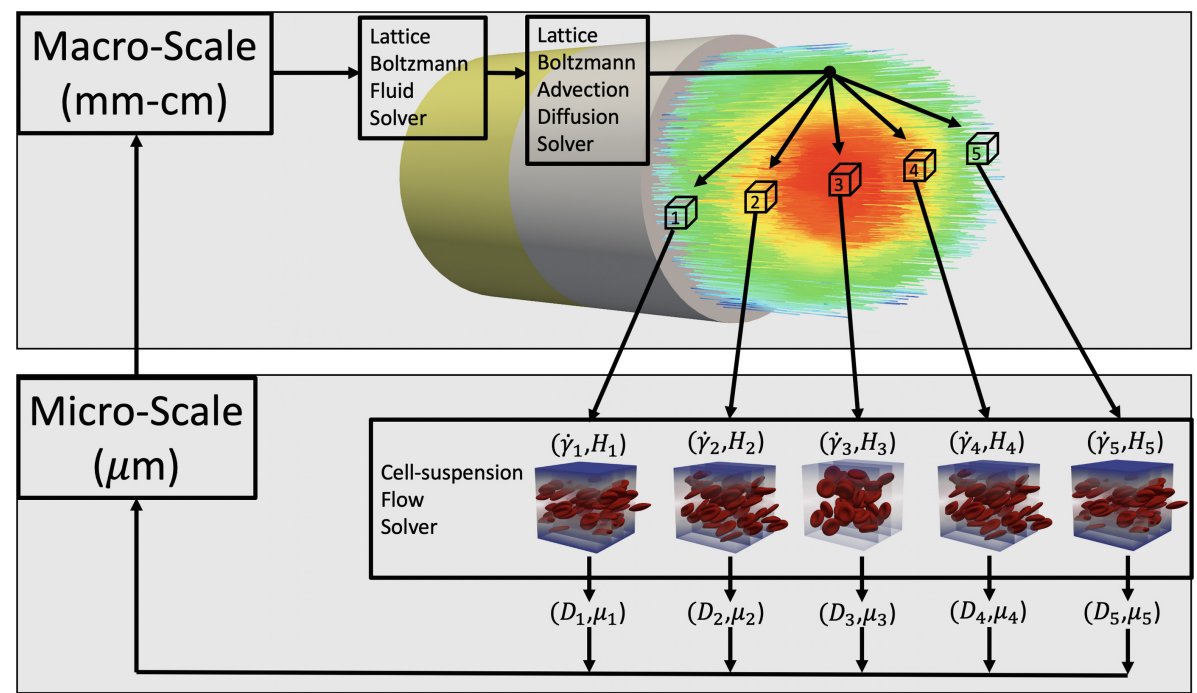

Fig. 1. Schematic highlighting the physical scales and types of numerical models employed in the HMM model.

A benefit of such an HMM model applied to blood flow is that on the largest scales a continuous blood flow solver will be informed by a micro scale cell resolved blood flow solver, resolving the cell nature of whole blood by keeping 
computational overhead in mind. The heterogeneity of the models allows for extension to be added, such as the diffusion of chemicals. A schematic highlighting the different numerical models and physical scales present in the HMM model is shown in Fig. 1, and a flow chart of the operations through one time-step of this model is highlighted in Fig. 2.

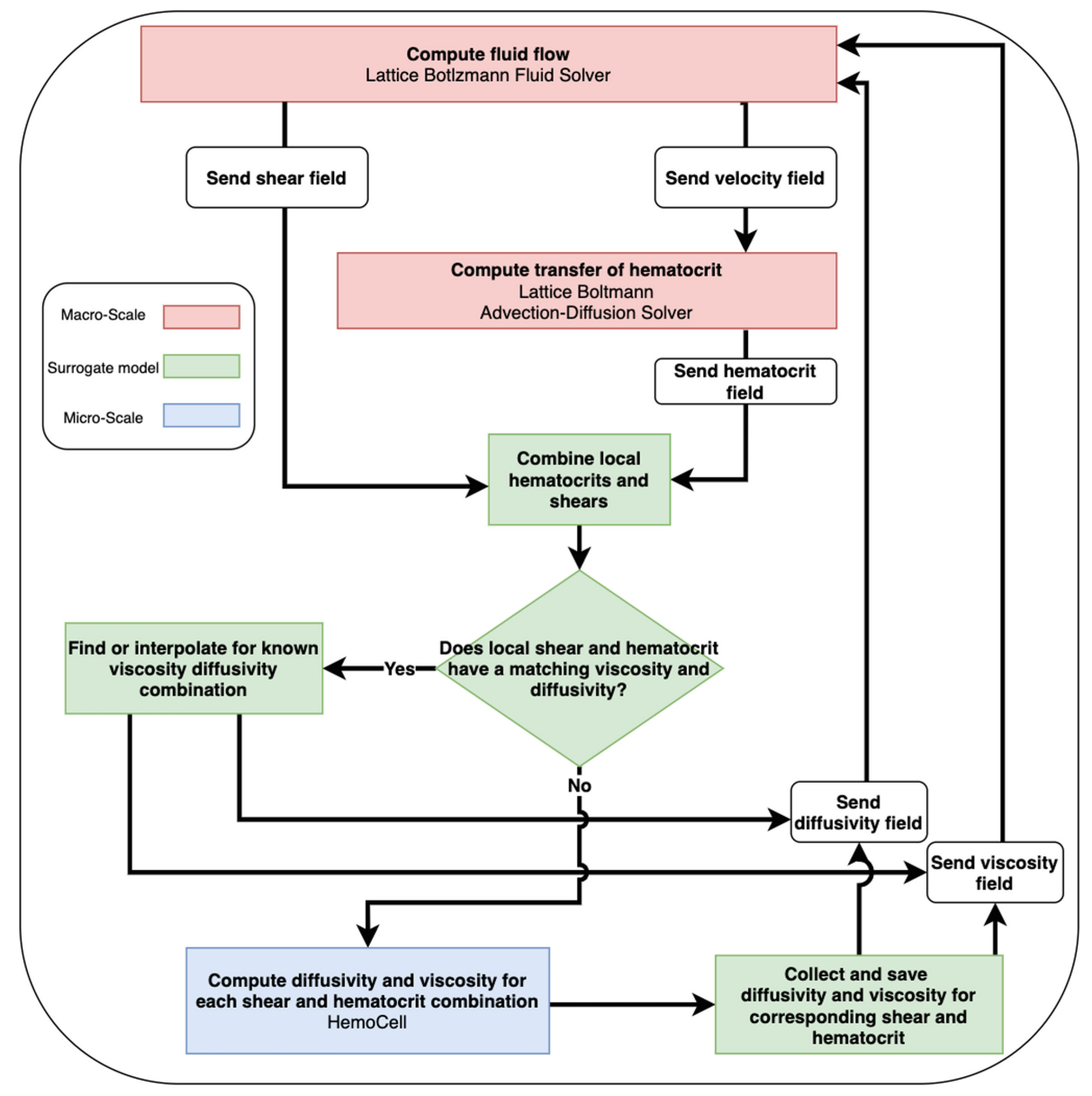

Fig. 2. Workflow of the HMM blood flow model.

\subsection{Macro Model}

On the largest scales in this model (Macro-scale) blood flow is modelled as a continuous fluid using the lattice Boltzmann method (LBM) [7]. Fluid will be driven by an external body force through a straight vessel geometry. After a time-step of the LBM fluid solver the velocity field across the macroscopic 
domain will be passed as input to an LBM advection-diffusion solver, which is also on the macro scale and will compute the transport of RBCs (hematocrit) given the underlying fluid flow. After this step the Hematocrit field from the advection diffusion solver and the velocity field (shear rate) from the fluid solver will be sent to a surrogate model. Here the surrogate model it will determine, via interpolation, whether a viscosity and diffusion coefficient is known for the shear rate and hematocrit combination. If there is no such parameter, either viscosity or diffusion coefficient, then a cell resolved blood flow model is spawned which will compute the diffusion coefficient and viscosity directly. The macro scale model will wait for either the surrogate model or the micro-scale model to return viscosities and diffusion coefficients for the entire domain. The viscosity field will be return to the fluid solver and the diffusion field will be returned to the advection diffusion solver. The time-step will then be incremented and the process will begin again.

\subsection{Coupling Between Micro and Macro}

To avoid duplicating shear rate and local hematocrit micro-scale simulations for each iteration of the HMM, we aim to build a surrogate model. This process will decrease the required number of micro-scale models requested, which in turn will decrease computation time. Hematocrit fields and shear rate fields will be passed down from the macro scale. First local hematocrit values and shear rate values will be combined, then the surrogate will query a database if there already exists a known viscosity and diffusion coefficient for the combination of hematocrit and shear rate. The surrogate will also conduct interpolations (based on a Gaussian process) for similar parameters. If the is a known viscosity and diffusion coefficients then it will return them to the macro scale. If there are missing viscosities and/or diffusion coefficients then a micro model will be spawned for each unknown shear rate and hematocrit combination. A heterogeneous multiscale computing framework has recently been developed to handle the scheduling and sub-model synchronization of such an application in order to efficiently use computational resources [4].

\subsection{Micro Model}

The micro scale is modelled with the cell resolved blood flow model HemoCell, in which plasma is modelled by the LBM, the mechanical model of the RBCs are described by a discrete element method and are couple to the plasma via the immersed boundary method $[26,27]$. From each local hematocrit and shear rate combination on the macro scale, a cell resolved micro scale will simulate perfect sheared environments using Lees-Edwards boundary conditions [21]. A single simulation will be spawned for each unknown diffusivity and shear rate pair, this will likely result in multiple spawns if there are multiple unknown input parameters. The resulting diffusion coefficients and viscosities will be computed from each cell resolved simulation. After each model has finished the results will be stored into the surrogate model and passed up towards the macro scale. As 
the HMM model progresses through time however a smaller amount of micro model will be spawned due to the surrogate model.

\section{Discussion}

The immediate focus of this work is to implement the HMM model for blood flow in a 3D straight vessel. Validation to known blood quantities such as the Fåhraeus-Lindqvist effect is needed. A correct physical implementation of this model will allow the determination of the minimum spatial size required from the cell resolved micro models, in order to preserve accurate viscosity and diffusivity measurements that will maximize computational efficiency. The determination of the minimum spatial resolution of local viscosity and diffusivity is also required on the macro scale in order to accurately and efficiently model blood flow.

The novelty of developing an HMM model for blood flow is that it can be extended to include models that are not limited to only model fluid dynamics. Given the heterogeneous nature of this model, computational models which include the reaction and perfusion of chemical species in the human microbiome, as well as the models for the smooth muscle cells, for example, can also be included to contribute to a more complete HMM model for the physiological human.

\section{References}

1. Abdulle, A., Weinan, E., Engquist, B., Vanden-Eijnden, E.: The heterogeneous multiscale method. Acta Numer. 21, 1-87 (2012). https://doi.org/10.1017/ S0962492912000025

2. Abraham, F., Behr, M., Heinkenschloss, M.: Shape optimization in steady blood flow: a numerical study of non-newtonian effects. Comput. Methods Biomech. Biomed. Eng. 8(2), 127-137 (2005)

3. Alowayyed, S., Závodszky, G., Azizi, V., Hoekstra, A.G.: Load balancing of parallel cell-based blood flow simulations. J. Comput. Sci. 24, 1-7 (2018). https://doi.org/ 10.1016/j.jocs.2017.11.008

4. Alowayyed, S.A., Vassaux, M., Czaja, B., Coveney, P.V., Hoekstra, A.G.: Towards heterogeneous multi-scale computing on large scale parallel supercomputers. Supercomputing Front. Innov. 6(4), 20-43 (2020)

5. Bagchi, P.: Mesoscale simulation of blood flow in small vessels. Biophys. J. 92(6), 1858-1877 (2007)

6. Barshtein, G., Wajnblum, D., Yedgar, S.: Kinetics of linear rouleaux formation studied by visual monitoring of red cell dynamic organization. Biophys. J. 78(5), 2470-2474 (2000)

7. Chen, S., Doolen, G.D.: Lattice boltzmann method for fluid flows. Annu. Rev. Fluid Mech. 30(1), 329-364 (1998)

8. Cheng, L.T., Weinan, E.: The heterogeneous multi-scale method for interface dynamics. Contempo. Math. 330, 43-54 (2003)

9. Chien, S., Usami, S., Taylor, H.M., Lundberg, J.L., Gregersen, M.I.: Effects of hematocrit and plasma proteins on human blood rheology at low shear rates. J. Appl. Physiol. 21(1), 81-87 (1966) 
10. Cokelet, G.R., Goldsmith, H.L.: Decreased hydrodynamic resistance in the twophase flow of blood through small vertical tubes at low flow rates. Circ. Res. 68(1), 1-17 (1991)

11. Dintenfass, L.: Internal viscosity of the red cell and a blood viscosity equation. Nature 219(5157), 956-958 (1968)

12. Weinan, E., Engquist, B.: The heterognous multiscale methods. Commun. Math. Sci. 1(1), 87-132 (2003). https://projecteuclid.org:443/euclid.cms/1118150402

13. Fåhræus, R., Lindqvist, T.: The viscosity of the blood in narrow capillary tubes. Am. J. Physiol. Legacy Content 96(3), 562-568 (1931)

14. Fedosov, D.A., Caswell, B., Popel, A.S., Karniadakis, G.E.: Blood flow and cell-free layer in microvessels. Microcirculation 17(8), 615-628 (2010)

15. Fedosov, D.A., Fornleitner, J., Gompper, G.: Margination of white blood cells in microcapillary flow. Phys. Rev. Lett. 108(2), 028104 (2012)

16. Fischer, T.M., Stohr-Lissen, M., Schmid-Schonbein, H.: The red cell as a fluid droplet: tank tread-like motion of the human erythrocyte membrane in shear flow. Science 202(4370), 894-896 (1978)

17. Hoekstra, A.G., et al.: Towards the virtual artery: a multiscale model for vascular physiology at the physics-chemistry-biology interface. Philos. Trans. Royal Soc. A Math. Phys. Eng. Sci. 374(2080), 20160146 (2016)

18. Hoekstra, A.G., van Bavel, E., Siebes, M., Gijsen, F., Geris, L.: Virtual physiological human 2016: translating the virtual physiological human to the clinic (2018)

19. Jung, J., et al.: Reference intervals for whole blood viscosity using the analytical performance-evaluated scanning capillary tube viscometer. Clin. Biochem. 47(6), 489-493 (2014)

20. Latt, J.: Palabos, parallel lattice boltzmann solver (2009)

21. Lorenz, E., Hoekstra, A.G., Caiazzo, A.: Lees-edwards boundary conditions for lattice boltzmann suspension simulations. Phys. Rev. E 79(3), 036706 (2009)

22. Nadeem, S., Akbar, N.S., Hendi, A.A., Hayat, T.: Power law fluid model for blood flow through a tapered artery with a stenosis. Appl. Math. Comput. 217(17), 7108-7116 (2011)

23. Tarksalooyeh, V.A., Závodszky, G., Hoekstra, A.G.: Optimizing parallel performance of the cell based blood flow simulation software hemocell. In: Rodrigues, J., et al. (eds.) ICCS 2019. LNCS, vol. 11538, pp. 537-547. Springer, Cham (2019). https://doi.org/10.1007/978-3-030-22744-9_42

24. Updegrove, A., Wilson, N.M., Merkow, J., Lan, H., Marsden, A.L., Shadden, S.C.: Simvascular: an open source pipeline for cardiovascular simulation. Ann. Biomed. Eng. 45(3), 525-541 (2017)

25. Závodszky, G., Paál, G.: Validation of a lattice boltzmann method implementation for a 3D transient fluid flow in an intracranial aneurysm geometry. Int. J. Heat Fluid Flow 44, 276-283 (2013)

26. Zavodszky, G., van Rooij, B., Azizi, V., Alowayyed, S., Hoekstra, A.: Hemocell: a high-performance microscopic cellular library. Procedia Comput. Sci. 108, 159-165 (2017)

27. Závodszky, G., van Rooij, B., Azizi, V., Hoekstra, A.: Cellular level in-silico modeling of blood rheology with an improved material model for red blood cells. Front. Physiol. 8, 563 (2017)

28. Závodszky, G., van Rooij, B., Czaja, B., Azizi, V., de Kanter, D., Hoekstra, A.G.: Red blood cell and platelet diffusivity and margination in the presence of crossstream gradients in blood flows. Phys. Fluids 31(3), 031903 (2019) 